\title{
Agradecimento aos Revisores da RBMFC em 2021
}

Acknowledgment of all those who reviewed for RBMFC in 2021

Reconocimiento a los revisores de la RBMFC en 2021

Claunara Schilling Mendonça ${ }^{1}$, Patricia Sampaio Chueiri ${ }^{1}$ Thiago Dias Sarti

${ }^{1}$ Revista Brasileira de Medicina de Família e Comunidade (RBMFC)

A Revista Brasileira de Medicina de Família e Comunidade (RBMFC) agradece aos Avaliadores listados abaixo que atuaram como revisores ad hoc durante o ano de 2021, dedicando horas voluntariamente para a emissão de pareceres técnicos sobre manuscritos submetidos a esta revista.

1. Adelson Jantsch, Fundação para o Desenvolvimento Científico e Tecnológico em Saúde. Rio de Janeiro, RJ.

2. Airton Tetelbom Stein.Universidade Federal de Ciências da Saúde de Porto Alegre (UFCSPA). Porto Alegre, RS

3. Alberto Novaes Ramos Jr. Universidade Federal do Ceará (UFC), Fortaleza, CE

4. Alessandra Martins dos Reis. Universidade do Estado da Bahia (UNEB). Salvador, BA.

5. Alessandro da Silva Scholze. Universidade do Vale do Itajaí (UNIVALI), Itajaí, SC.

6. Alexandre José de Melo Neto. Universidade Federal da Paraíba (UFPB). João Pessoa, PB.

7. Alisson Oliveira dos Santos. Universidade Federal de Ouro Preto / Universidade Federal de São João del-Rei / Universidade do Estado do Rio de Janeiro.

8. Almária Mariz Batista. Universidade Federal do Rio Grande do Norte (UFRN). Escola Multicampi de Ciências Médicas do Rio Grande do Norte. Caicó, RN.

9. Ana Cláudia Santos Chazan. Universidade do Estado do Rio de Janeiro (UERJ). Rio de Janeiro, RJ.

10. Armando Henrique Norman. Universidade Federal de Santa Catarina (UFSC), Florianópolis, SC

11. Bruna Moretti Luchesi. Universidade Federal de Mato Grosso do Sul (UFMS). Campus de Três Lagoas, MS

12. Bruna Pontes Silva. Escola de Saúde Pública de Florianópolis. Florianópolis, SC.

13. Bruno José Barcellos Fontanella. Universidade Federal de São Carlos (UFSCar). São Carlos, São Paulo

14. Bruno Pereira Stelet. Escola Nacional de Saúde Pública FIOCRUZ. Secretaria de Saúde do Distrito Federal. Universidade do Estado do Rio de Janeiro.

Como citar: Mendonça CS, Chueiri PS, Sarti TD. Agradecimento aos Revisores da Revista Brasileira de Medicina de Família e Comunidade (RBMFC) que avaliaram manuscritos em 2021. Rev Bras Med Fam Comunidade. 2021;16(43):3351 http://doi.org/10.5712/rbmfc16(43)3351 
15. Camila Carvalho de Souza Amorim Matos. Universidade Federal de Santa Catarina (UFSC). Florianópolis, SC.

16. Carine Oliveira. Universidade Federal do Recôncavo da Bahia. (UFRB). Cruz das Almas, BA.

17. Carlos André Aita Schmitz. Universidade Federal do Rio Grande do Sul (UFRGS), Porto Alegre, RS.

18. Carlos Frederico Confort Campos. Universidade de São Paulo (USP). São Paulo, SP.

19. Carolina Maria do Carmo Alonso. Universidade Federal do Rio de Janeiro (UFRJ), Rio de Janeiro, RJ.

20. Cássio de Almeida Lima. Universidade Estadual de Montes Claros (Unimontes), Montes Claros, MG.

21. Célia Aparecida Paulino. Ex-UNIAN/SP (aposentada)

22. Clécio Homrich da Silva. Universidade Federal do Rio Grande do Sul (UFRGS). Porto Alegre, RS.

23. Cristine Vieira do Bonfim. Fundação Joaquim Nabuco (FUNDAJ). Recife, Pernambuco

24. Daniel Almeida Gonçalves. Universidade Federal de São Paulo (UNIFESP), São Paulo, SP

25. Daniel Knupp Augusto, Sociedade Brasileira de Medicina de Família e Comunidade (SBMFC), Rio de Janeiro, RJ.

26. Daniel Moreira Pinto. Universidade Federal dos Vales do Jequitinhonha e Mucuri (UFVJM). Teófilo Otoni, MG.

27. David Ramos da Silva Rios. Universidade Federal da Bahia (FMB/UFBA). Salvador, BA.

28. Debora Carvalho Ferreira. Universidade Federal de Viçosa (UFV). Viçosa, MG.

29. Débora Cristina Modesto Barbosa. Universidade de Franca (UNIFRAN), Franca, SP.

30. Débora Teixeira. Universidade do Estado do Rio de Janeiro (UERJ). Rio de Janeiro, RJ.

31. Deise Fernanda Peixoto Oliveira. Universidade Federal de Sergipe (UFS). São Cristóvão, SE.

32. Diana de Oliveira Frauches. Escola Superior de Ciências da Santa Casa de Misericórdia de Vitória. Vitória, ES

33. Diani Oliveira Machado. Universidade Federal do Rio Grande do Sul (UFRGS). Porto Alegre, RS.

34. Diego José Brandão. Universidade Vila Velha (UVV). Vila Velha, ES

35. Donovan Casas Patiño, Universidad Autonoma del Estado de Mexico (UAEM) [Centro Universitario Amecameca], México.

36. Eduardo Alexander Júlio César Fonseca Lucas. Universidade Federal do Rio de Janeiro (UFRJ). Rio de Janeiro, RJ.

37. Eduardo Bertol. Secretaria Municipal de Saúde de Curitiba, Curitiba, PR.

38. Efigenia Ferreira. Universidade Federal de Minas Gerais (UFMG), Belo Horizonte, MG.

39. Elisabeth Meloni Vieira. Universidade de São Paulo (USP), São Paulo, SP.

40. Fatima Aparecida Ferreira Teixeira de Carvalho. Universidade de Ribeirão Preto (UNAERP). Ribeirão Preto, SP

41. Felipe Proenço. Universidade Federal da Paraíba (UFPB). João Pessoa, PB.

42. Ferla Maria Simas Bastos Cirino. Prefeitura Municipal de Diadema (PM Diadema). Diadema, SP.

43. Fernanda Pereira de Paula Freitas. Universidade Federal do Rio de Janeiro (UFRJ). Macaé, RJ.

44. Francisco Arsego de Oliveira. Universidade Federal do Rio Grande do Sul (UFRGS). Porto Alegre, RS

45. Fúlvio Borges Nedel. Rede de Pesquisa em Atenção Primária à Saúde (REDE APS).

46. Giuliano Dimarzio. Sociedade Brasileira de Medicina de Família e Comunidade (SBMFC). Rio de Janeiro, RJ.

47. Gustavo Diniz Ferreira Gusso. Universidade de São Paulo (USP). São Paulo, SP

48. Hilmar Dias Ricardo. Universidade do Estado do Rio de Janeiro (UERJ). Rio de Janeiro, RJ.

49. Isabel Brandão Correia. Secretaria de Saúde do Recife. Recife, PE. 
50. Jardel Corrêa de Oliveira. Prefeitura Municipal de Florianópolis (PMF), Florianópolis, SC

51. João Werner Falk, Universidade Federal do Rio Grande do Sul (UFRGS). Porto Alegre, RS

52. Jocinei Santos de Arruda. Universidade do Vale do Rio dos Sinos ( UNISINOS). São Leopoldo, RS

53. Johnnatas Mikael Lopes. Universidade Federal do Vale do São Francisco (UNIVASF). Petrolina, PE.

54. Josenaide Engracia Santos. Universidade de Brasília (UNB). Brasília, DF.

55. Juliana da Rosa Wendt. Universidade Federal de Santa Maria (UFSM). Santa Maria, RS.

56. Julie Silvia Martins. Atenção Primária à Saúde Santa Marcelina (APS Santa Marcelina). Itaquera, SP.

57. Leandro David Wenceslau. Universidade Federal de Viçosa (UFV). Viçosa, Minas Gerais.

58. Leonardo Ferreira Fontenelle. Universidade Vila Velha (UVV). Vila Velha, ES.

59. Leonardo Vieira Targa, Universidade de Caxias do Sul (UCS). Caxias do Sul, RS

60. Lourdes Luzón Oliver. Servicio Castellano Manchego de Salud, Gerencia de Atención Integrada de Talavera de la Reina. Talavera de la Reina, Toledo.

61. Lucas Alexandre Pedebôs. Prefeitura Municipal de Florianópolis (PMF), Florianópolis, SC.

62. Lucas Mello Pioner, Universidade Federal de Santa Catarina (UFSC), Florianópolis, SC

63. Lucas Wollmann. Grupo Hospitalar Conceição (GHC). Porto Alegre, RS.

64. Luciana Tricai Cavalini. Secretaria de Estado da Saúde de Santa Catarina (SES-SC). Florianópolis, SC.

65. Luisa da Matta Machado Fernandes. Instituto Rene Rachou / FIOCRUZ Minas. Belo Horizonte, MG

66. Luiz Paulo Gomes dos Santos Rosa. Universidade Federal do Rio Grande do Norte (UFRN). Natal, RN.

67. Maiara Conzatti, Universidade Federal do Rio Grande do Sul (UFRGS). Porto Alegre, RS.

68. Marcello Dala Bernardina, Secretaria de Estado da Saúde do Espírito Santo (SESA) e Hospital Universitário Cassiano Antonio de Moraes (HUCAM). Vitória, ES

69. Marcelo Pellizzaro Dias Afonso. Universidade Federal de Minas Gerais (UFMG), Belo Horizonte, MG.

70. Marcelo Rodrigues Gonçalves. Universidade Federal do Rio Grande do Sul (UFRGS). Porto Alegre, RS

71. Marcos Antonio Barbosa Pacheco, Secretaria Municipal de Saúde (São Luis, MA); Universidade CEUMA. São Luis, MA

72. Margarita Silva Diercks. Hospital Nossa Senhora da Conceição-GHC (CEPAPS-SSC-GHC). Porto Alegre, RS.

73. Mariana Felgueira Pavanelli. Centro Universitário Integrado. Campo Mourão, PR.

74. Marília Jesus Batista. Faculdade de Medicina de Jundiaí. Jundiaí, SP.

75. Mário Roberto Tavares Cardoso de Albuquerque. Centro Univerversitário do Pará (CESUPA). Belém, PA

76. Matheus Alves Alvares. Centro Universitário Lusíada. Santos, SP.

77. Maurício Moraes. Universidade Federal de Rio Grande (UFRGS). Porto Alegre, RS.

78. Melanie Noël Maia. Universidade Federal do Rio de Janeiro (UFRJ), Rio de Janeiro, RJ.

79. Mellina Izecksohn. Ensp/Fiocruz e Universidade Federal do Rio de Janeiro (UFRJ). Rio de Janeiro, RJ.

80. Murilo Moura Sarno. Universidade Municipal de São Caetano do Sul. (USCS). São Caetano, SP.

81. Nicole Geovana Dias Carneiro. Universidad de Alicante.

82. Nivaldo Carneiro Junior. Faculdade de Ciências Médicas da Santa Casa de São Paulo. São Paulo, SP.

83. Olivia Ferreira Lucena. Universidade Federal da Paraíba (UFPB). João Pessoa, PB.

84. Pablo de Lannoy Stürmer. Grupo Hospitalar Conceição (GHC). Porto Alegre, RS.

85. Patricia Barreto Cavalcanti. Universidade Federal da Paraíba (UFPB). João Pessoa, PB

86. Patrícia Silveira Rodrigues. Universidade Estadual de Campinas (UNICAMP). Campinas, SP.

87. Pedro Walisson Gomes Feitosa. Universidade Federal do Cariri (UFCA). Juazeiro do Norte, CE.

88. Rafaela Cordeiro Freire. Universidade Federal da Bahia (UFBA), Salvador, BA. 
89. Raquel Ferreira, Instituto René Rachou. Fundação Oswaldo Cruz. Rio de Janeiro, RJ.

90. Ricardo de Mattos Russo Rafael. Universidade do Estado do Rio de Janeiro (UERJ) / Universidade Estácio de Sá (UNESA), Rio de Janeiro, RJ.

91. Rinaldo Eduardo Machado de Oliveira. Faculdade de Medicina de Ribeirão Preto da Universidade de São Paulo (FMRP-USP). São Paulo, SP.

92. Roberta Reis. Universidade Federal do Rio Grande do Sul (UFRGS). Porto Alegre, RS

93. Roberto Henrique Amorim de Medeiros. Universidade Federal do Rio Grande do Sul (UFRGS), Porto Alegre, RS.

94. Rodolfo Rêgo Deusdará Rodrigues. Universidade de Brasília (UnB), Brasília, DF.

95. Rodrigo Caprio Leite de Castro. Universidade Federal do Rio Grande do Sul (UFRGS). Porto Alegre, RS, Brasil

96. Ronaldo da Silva Cruz. Faculdade Cathedral. Boa Vista RR

97. Rosália Garcia Neves. Universidade Federal de Pelotas (UFPel). Pelotas, RS.

98. Rosalva Grobério Pazó. Universidade Federal do Espírito Santo (UFES), Vila Velha, ES.

99. Rosana Moysés. Universidade Federal do Amazonas (UFAM). Manaus, AM.

100. Sergio Antonio Sirena. Grupo Hospitalar Conceição (GHC) Fundação Universidade de Caxias do Sul, Caxias do Sul, RS

101. Sérgio Henrique de Oliveira Botti. Universidade Federal do Estado do Rio de Janeiro (UNIRIO), Rio de Janeiro, RJ.

102. Sonia Maria Martins. Sociedade Brasileira de Medicina de Familia e Comunidade. (SBMFC), Rio de Janeiro, RJ.

103. Tãnia de Araujo Barboza. Universidade de Fortaleza (UNIFOR), Fortaleza, CE

104. Thiago Santos Souza. Universidade Federal da Bahia (UFBA). Instituto de Saúde Coletiva (ISC). Salvador, BA.

105. Welington Serra Lazarini. Universidade Federal do Espírito Santo (UFES). Vitória, ES.

106. Yuji Magalhães Ikuta. Universidade do Estado do Pará (UEPA) / Universidade Federal do Pará (UFPA). Belém, PA, Brasil. 